\title{
Non-alcoholic fatty liver disease: controlling an emerging epidemic, challenges, and future directions
}

\author{
Andrew Ofosua, Daryl Ramaia, ${ }^{\mathrm{a}, \mathrm{b}}$, Madhavi Reddy ${ }^{\mathrm{a}}$
}

The Brooklyn Hospital Center Clinical Affiliate of the Mount Sinai Hospital, Brooklyn; St. George’s University, True Blue, Grenada, WI

\begin{abstract}
Non-alcoholic fatty liver disease (NAFLD) affects over 30\% of the United States population and is projected to become a leading cause of chronic liver disease by 2020. As a result, the economic and societal burden of NAFLD is far-reaching. The cost of managing NAFLD complications has an estimated 10 year economic burden of $\$ 908$ billion. This review provides an overview of current knowledge on NAFLD, with emphasis on identifying gaps in its diagnosis and management, and proposes future directions to address these limitations. Despite the increasing prevalence of NAFLD, there is limited knowledge and practice regarding its natural history, staging, diagnosis, and management. Though a challenging task, opportunities for bridging these gaps should focus on the development of noninvasive biomarkers, the elucidation of biological pathways, the creation of up-to-date screening guidelines, and the organization of clinical trials of longer duration to determine clinical endpoints and assess the safety of new treatment options.
\end{abstract}

Keywords Non-alcoholic fatty liver disease, non-alcoholic steatohepatitis, liver transplantation, cirrhosis

Ann Gastroenterol 2018; 31 (3): 1-8

\section{Introduction}

Non-alcoholic liver disease is histologically similar to alcoholic liver disease but without a history of alcohol consumption. Nonalcoholic fatty liver disease (NAFLD) represents a spectrum of disease from non-alcoholic fatty liver (NAFL), characterized by hepatic fat accumulation without inflammation, to non-alcoholic steatohepatitis (NASH), characterized by hepatic fat deposition with inflammation, accumulating fibrosis, and ultimately liver cirrhosis [1]. NASH-related cirrhosis is currently a leading cause of chronic liver disease and is associated with hepatocellular cancer. It has emerged as the second leading indication for liver transplant evaluation in the United States [2,3].

NAFLD is a leading cause of liver-related morbidity and mortality; the literature shows that it is also associated with

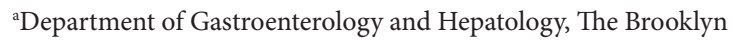
Hospital Center Clinical Affiliate of the Mount Sinai Hospital, Brooklyn (Andrew Ofosu, Daryl Ramai, Madhavi Reddy); 'SChool of Medicine, St. George's University, True Blue, Grenada (Daryl Ramai), WI

\section{Conflict of Interest: None}

Correspondence to: Andrew Ofosu, MD, MPH, Department of Gastroenterology and Hepatology, The Brooklyn Hospital Center, Clinical Affiliate of the Mount Sinai Hospital Brooklyn, NY 11201, USA, e-mail: aofosu@tbh.org

Received 16 October 2017; accepted 22 January 2017; published online 23 February 2018

DOI: https://doi.org/10.20524/aog.2018.0240 increased overall mortality from cardiovascular causes, increased incidence of type 2 diabetes, and increased risk of chronic kidney diseases [4,5]. Agarwal et al reported a cross-sectional study that identified NAFLD as an independent predictor of coronary artery disease, hypertension, and elevated low-density lipoprotein cholesterol [6]. Risk factors related to NAFLD include increasing age, obesity, insulin resistance, and small nucleotide polymorphisms in two genes, PNPLA3 (encoding patatin-like phospholipase domain-containing protein 3) and TM6SF2 (encoding transmembrane 6 superfamily member 2) [7].

Despite the alarming rate of non-alcoholic liver disease, there are limitations in knowledge and unmet needs in the management of NAFLD among medical providers. In a survey conducted among primary care physicians, 58\% expressed a lack of confidence in their knowledge and management of fatty liver disease [8]. The challenge in diagnosing NAFLD may stem from the fact that most patients are asymptomatic and are typically only identified by routine blood tests showing elevated liver enzymes. Nevertheless, a subset of patients can have normal liver enzymes and thus remain undiagnosed.

Numerous studies have looked at the management of fatty liver disease; however, there is no consensus on the optimal management of NAFLD. Currently, there are no drug therapies approved by the Food and Drug Association (FDA) for the treatment of NAFLD. This article aims to review current knowledge on NAFLD, with emphasis on identifying gaps in its diagnosis and management, and proposes future directions to address these limitations. 


\section{Methodology}

Using PubMed, MEDLINE, CINAHL, EMBASE, Scopus, and Cochrane Databases, we searched for articles using the keywords "Non-alcoholic fatty liver disease", "Non-alcoholic steatohepatitis," "Fatty liver," "Liver fibrosis," "Cirrhosis," and "Chronic liver disease" between 2005 and 2017. Articles published in languages other than English were excluded. Original articles, case-control trials, clinical reviews, metaanalyses, randomized trials and clinical guidelines were reviewed.

\section{Epidemiology of NAFLD}

NAFLD is currently the most common liver disorder, particularly in Western countries. Worldwide, the prevalence of NAFLD is about $25 \%$, with the highest rates reported in South America (31\%) and the Middle East (32\%), followed by Asia (27\%), the USA (24\%) and Europe (23\%); NAFLD is less common in Africa (14\%) [9]. Reports from the National Health and Nutrition Examination Survey have shown that the prevalence of fatty liver disease in patients with chronic liver disease increased from $47 \%$ to $75 \%$ between 1999 and 2008 [10]. Overall, the prevalence of NAFLD is increasing, particularly in the United States, and it has been projected to become a leading cause of chronic liver disease by 2020 [2].

The prevalence of NAFLD tends to parallel that of high risk groups with metabolic syndrome, though NAFLD can occur in patients with a normal basic metabolic index. A high prevalence of comorbid conditions in patients with NAFLD, and vice versa, have long established a directional causal relationship that warrants future investigation [11]. Among patients with type two diabetes the prevalence of NAFLD ranges from $33 \%$ to $66 \%$, while the prevalence of diabetes among patients with NAFLD has been reported to be $43 \%$ [9,12-15]. Among patients with obesity, the prevalence of NAFLD can exceed 95\% [16,17]. Additionally, other comorbid conditions have been reported among patients with NAFLD, including hyperlipidemia (69\%), hypertriglyceridemia (41\%), metabolic syndrome (43\%), and hypertension (39\%) [9].

The majority of patients diagnosed with NAFLD are in their 40s or 50s; however, there are conflicting data with regard to sex distribution [2]. A population-based study found the prevalence of NAFLD/NASH to be higher in Hispanics (58.3\%) compared to whites (44\%) and blacks (35.1\%) [18] (Fig. 1). The difference in racial and ethnic prevalence may be dependent on an interplay of socioeconomic, behavioral and genetic factors. However, while the prevalence of NAFLD may vary with sex and ethnicity, increasing age has been associated with the occurrence and stage of liver disease [19].

\section{Clinical and economic burden of fatty liver disease}

Using prediction models, Younossi et al reported that the annual clinical burden of NAFLD is expected to increase, with over 12 million cases of NAFL and over 600,000 cases of NASH predicted [20]. Similar trends in annual economic burden are predicted to affect Europe and the US with over $\$ 103$ billion per year in direct cost. A further increase in the cost of managing disease-related complications is estimated to bring the 10-year economic burden of NAFLD as high as $\$ 908$ billion.

The annual cost is further compounded by societal cost due to the loss of quality-adjusted life years as a result of NAFLD and resulting complications [20]. Incorporating societal loss leads to an estimated value of $\$ 292.2$ billion. The economic burden is predicted to rise as the clinical consequences of NAFLD increase. It is worth noting that the cost of treatment was not accounted for in these models.

Medicare data in patients with NAFLD have shown that the mean yearly inflation-adjusted value in outpatient care increased from $\$ 2624 \pm \$ 3308$ in 2005 to $\$ 3608 \pm \$ 5132$ in 2010 , with a median total hospital charge of $\$ 36,289$ in 2010 [20,21]. The current trajectory results in Medicare patients with NAFLD utilizing an enormous amount of inpatient and outpatient care resources [22].

\section{Clinical course}

Outcomes of patients with NAFLD have been studied before; however, previous studies were limited by the number of patients and short follow-up times. Overall, NAFL without steatosis progresses slowly, unlike NASH, which has a greater potential to progress to cirrhosis and its related complications. Approximately $30 \%$ of patients with NAFLD develop NASH [23]. About 20-40\% of patients with NASH are likely to develop progressive liver fibrosis [3]. In less than 5\% of NASH patients, fibrosis progresses to cirrhosis [24]. However, there are limited prospective data evaluating the progression of NAFLD to hepatocellular carcinoma (HCC). Ekstedt et al, in a follow up of patients with biopsy-proven NAFLD over a period of 26.4 years, observed a mortality rate of $5 \%$ due to HCC [25]. White et al, in a systematic review of patients with NAFLD and NASH, showed that the cumulative incidence of HCC ranged from $2.4 \%$ over 7 years to $12.8 \%$ over a period of 3 years [26]. A Veterans Affairs study by Mittal et al showed that HCC accounted for $8 \%$ of NAFLD cases [27].

The Prognostic Relevance of Liver Histology in NAFLD (PRELHIN) study has shed light on independent risk factors associated with death or liver transplantation among patients with NAFLD. Age, diabetes, and stage of fibrosis were independently associated with death or liver transplantation [28]. However, advanced fibrosis was the only known histological variable to be predictive of liver-related complications. A review of the literature indicates that fibrosis is the strongest predictor of mortality [23]. However, the natural history of NASH is heterogeneous and it remains difficult to predict which subset of patients are likely to progress. Large clinical trials with longer follow-up times accounting for comorbidities are needed to elucidate the details surrounding the natural history of the disease. 


\section{Barriers in controlling NAFLD}

\section{Who should be screened?}

Notwithstanding the gravity of the problem, along with the projected healthcare burden, multiple limitations and unmet needs in the management of NAFLD remain to be addressed. There are no clear screening guidelines for NAFLD. A survey conducted in Australia showed that non-hepatologists underestimated the prevalence of NAFLD, both in the general population and in highrisk patients [29]. The study also reported that only a few patients were referred to hepatologists. A similar trend was demonstrated in a French study showing that only $20 \%$ of NAFLD patients seen by a gastroenterologist were referred by other specialists [30]. Both studies showed that, when considering screening patients for NAFLD, there was an over-reliance on elevated liver enzymes, particularly elevated transaminases, instead of metabolic risk factors. Guidelines from the American Association for the Study of Liver Diseases (AASLD) do not recommend screening for NAFLD in family members or high-risk groups attending primary care, diabetic, or obesity clinics because of the lack of long-term benefits and cost-effectiveness [19].

Some hepatology societies recommend screening for NAFLD with liver function tests and ultrasound of the abdomen. The challenges with these diagnostic tests include the lack of standardization of ultrasound instruments in defining steatosis and the presence of normal transaminases in some patients with NAFLD, which does not correlate with histological disease [31]. Despite these challenges, the North American Society for Pediatric Gastroenterology, Hepatology and Nutrition (NASPGHAN) recently released guidelines recommending screening children aged 9-11 years with obesity and other children with risk factors using an alanine aminotransferase test [32]. The caveat, however, is that NAFLD can occur in non-obese patients without metabolic syndrome or type 2 diabetes mellitus.

Furthermore, according to the National Guideline Centre (UK), the use of routine liver function tests to diagnose NAFLD is ill-advised in children and young adults, and the Centre recommendsusingliverultrasoundinhigh-riskpatientsor patients suspected of having NAFLD [33]. Similar clinical guidelines have been issued by other European bodies [34]. Given these recent guidelines in the pediatric population, updated guidelines for adults are long overdue. Having a more standardized validated model that incorporates risk factors such as age, family history of NAFLD, metabolic syndrome, ethnicity, and sleep apnea will streamline the process of screening, especially among primary care providers. This approach will aid the earlier detection of NAFLD. Further research is need to define this model and determine screening intervals in high-risk patients.

\section{Diagnostic challenges}

Most patients with NAFLD are asymptomatic and are diagnosed following incidental laboratory findings. The diagnosis of NAFLD requires a combination of clinical history, serologic testing, and radiologic findings (ultrasonography, computer tomography scan, or magnetic resonance imaging) [35-37] (Table 1). Ultrasonography, though widely available, has limited diagnostic capacity. Computed tomography scanning, though more sensitive, entails a risk of exposure to radiation. In contrast, more

Table 1 Sensitivities and specificities of imaging modalities used to detect hepatic steatosis

\begin{tabular}{llcc}
\hline Modality & Severity & Sensitivity (\%) & Specificity (\%) \\
\hline US & Mild & $55-67$ & $77-93$ \\
US & Moderate-to-severe & $81-100$ & 98 \\
CT & Mild & 73 & 91 \\
CT & Moderate-to-severe & 82 & 100 \\
MRS & Overall & $80-91$ & $80-87$ \\
MRI & Overall & $77-90$ & $87-91$ \\
\hline
\end{tabular}

US, ultrasound; CT, computer tomography; MRS, magnetic resonance spectroscopy; MRI, magnetic resonance imaging

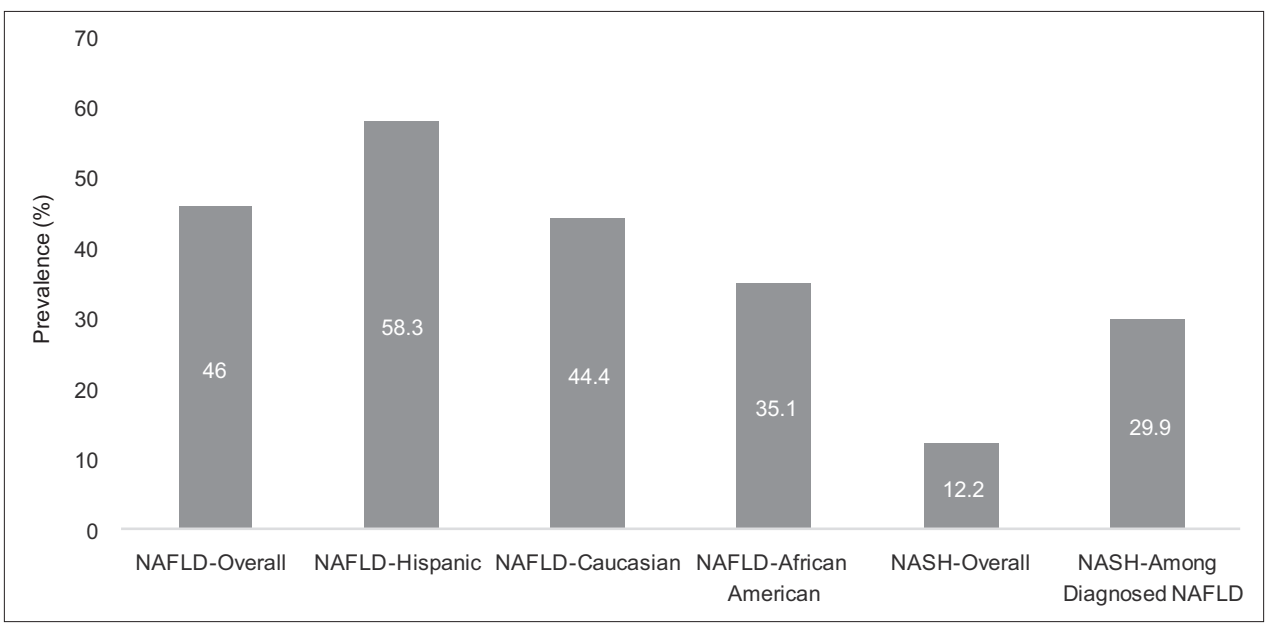

Figure 1. Prevalence of nonalcoholic fatty liver disease (NAFLD) and nonalcoholic steatohepatiits (NASH). Data updated from: Williams CD et al [19]. Used with the permission of Elsevier Inc. 
sensitive imaging modalities, such as magnetic resonance spectroscopy and magnetic resonance imaging, are expensive. Diagnosis is usually by exclusion, after secondary causes of fat accumulation have been ruled out $[33,34]$. Further research is required to develop cost-effective and less invasive modalities for diagnosing NAFLD.

\section{Disease staging}

After a diagnosis of NAFLD is made, it is imperative to determine the fibrotic stage of the disease. The extent of fibrosis has been shown to correlate with clinical outcomes. A prospective study involving patients with liver biopsies demonstrated that the presence and extent of fibrosis were the primary histological features of NAFLD that predicted decompensation and advanced disease [38]. There is growing interest in determining how to stage the extent of liver fibrosis noninvasively. The NAFLD fibrosis score (NFS), based on clinical data including age, body mass index, platelet count, and aspartate transaminase and alanine transaminase levels, has been validated as an effective biomarker in determining the extent of fibrosis [39]. Other noninvasive tools used to detect the presence of advanced fibrosis in NAFLD patients include the fibrosis-4 (FIB-4) score, aspartate-aminotransferaseto-platelet ratio, enhanced liver fibrosis panel, Fibrometer, FibroTest, and Hepascore [40]. A summary of clinical aids or biomarkers used to assess fibrosis can be seen in Table 2 . Overall, the AASLD recommends the use of the NFS or the FIB-4 index for identifying NAFLD patients with a higher likelihood of having bridging fibrosis or cirrhosis [19].

Imaging techniques that measure liver stiffness, such as transient elastography (TE) and magnetic resonance elastography (MRE), have emerged as being more accurate in predicting liver fibrosis. TE can have conflicting results in obese patients, primarily because subcutaneous fatty tissue attenuates elastic share wave, reducing diagnostic reliability [41]. Additionally, though TE represents a convenient and noninvasive test, approximately $25 \%$ of results are unreliable or uninterpretable [42]. MRE is superior to TE and is excellent for identifying varying degrees of fibrosis in patients with NAFLD [43]. MRE has a specificity of $0.91(95 \%$ confidence interval [CI] 0.83-0.96) and a sensitivity of 0.86 (95\%CI $0.65-0.97)$ in identifying patients with advanced fibrosis [44]. The AASLD recommends the use of TE and MRE for identifying advanced fibrosis in patients with NAFLD [19].

Liver biopsy remains the gold standard for confirming the diagnosis of NASH and evaluating inflammation or fibrosis. However, liver biopsy is limited by its invasiveness, cost and sampling error. The lack of an accurate, noninvasive method for distinguishing NAFL from NASH with fibrosis remains one of the unmet needs in the management of patients with NAFLD. Other diagnostic methods show promising results in the use of hepatic collagen fractional synthesis rate (FSR), and plasma FSR, which correlates with hepatic fibrosis in humans [44]. However, larger studies are required to validate these findings.

\section{Management}

\section{Challenges in non-pharmacological management}

The increasing incidence of NAFLD requires an amplified effort to detect the disease early in its course and reduce its progression. Diet and exercise are the mainstay of management in patients with NAFLD. In a prospective study of patients with biopsy-confirmed NASH, a $10 \%$ loss in body weight led to a histological benefit in improved fibrosis of the liver [45]. The benefit of weight loss has also been demonstrated in a trial that randomized dietician-reinforced lifestyle intervention versus general recommendations for weight loss. The study observed a $64 \%$ resolution in liver fibrosis in the intervention group compared with $20 \%$ in the control group [46]. A recent metaanalysis showed that exercise, alone or combined with dietary

Table 2 Biomarkers used to detect the presence of progressive liver fibrosis

\begin{tabular}{|c|c|c|c|c|}
\hline Test & Panel components & Sensitivity (\%) & Specificity (\%) & Fibrosis stage \\
\hline AST/ALT ratio & AST, ALT & 21 & 90 & F3-F4 \\
\hline AST/platelet ratio & AST, Platelet & 30 & 93 & $\mathrm{~F} 2-\mathrm{F} 4$ \\
\hline BAAT score & BMI, Age, ALT, Serum Triglycerides & 71 & 80 & $\mathrm{~F} 3-\mathrm{F} 4$ \\
\hline BARD score & BMI, AST/ALT, Diabetes & 87 & 33 & $\mathrm{~F} 3-\mathrm{F} 4$ \\
\hline ELF test & Age, HA, TIMP-1, PIIINP & 80 & 90 & F2-F4 \\
\hline Fibrometer & Platelet, G2 Macroglobulin, AST, Age, PT, HA, BUN & 81 & 84 & $\mathrm{~F} 2-\mathrm{F} 4$ \\
\hline Fibrotest & $\begin{array}{l}\text { a-2 Macroglobulin, Haptoglobin, GGT, Total Bilirubin, } \\
\text { Apolipoprotein }\end{array}$ & $15-77$ & $77-90$ & $\mathrm{~F} 2-\mathrm{F} 4$ \\
\hline FIB-4 Score & Age, AST, Platelet Count, ALT & $26-74$ & $71-98$ & $\mathrm{~F} 3-\mathrm{F} 4$ \\
\hline Hepascore & Age, Sex, Bilirubin, GGT, HA, a2-Macroglobulin & $76-87$ & $84-89$ & F3-F4 \\
\hline NAFLD fibrosis score & Age, Hyperglycemia, BMI, Platelet, Albumin, AST/ALT & 51 & 96 & F3-F4 \\
\hline
\end{tabular}

AST, aspartate aminotransferase; ALT, alanine aminotransferase; BMI, body mass index; HA, hyaluronic acid; GGT, $\gamma$-glutamyltransferase; PIIINP, procollagen III amino-terminal peptide; TIMP1, tissue inhibitor of matrix metalloproteinase 1; ELF, enhanced liver fibrosis; FIB-4, fibrosis-4; PT, prothrombin 
intervention, improves serum levels of liver enzymes and liver fat or histology in NAFLD patients [47]. Furthermore, resolution of liver fibrosis has therapeutic implications, since fibrosis has been shown to be a main predictor of disease progression and liver cirrhosis.

The ideal diet for patients is yet to be determined; however, certain lifestyle dietary habits have potential associations with NAFLD. A Mediterranean diet rich in polyunsaturated fatty acids and fiber during a 6-week crossover study resulted in a significant reduction in hepatic steatosis. It remains to be seen whether the short-term improvement in steatosis has longterm benefits in reducing disease progression [48]. A recent study reported that optimal sleep (defined as sleep hours $\geq 7$ and $\leq 9 /$ day) in addition to a diet comprising high consumption of low-fat dairy products, vegetables, and fish, was beneficially associated with insulin resistance and liver stiffness in NAFLD patients, independently of body weight status and energy intake [49]. Other studies have underscored the value of optimal sleep and its associSation with NAFLD [50].

A study comparing the effects of mild-, moderate- and high-intensity exercise regimens in patients with hepatic fat found that the highest exercise intensity program ( $>250 \mathrm{~min}$ per week) led to a significant reduction in hepatic fat [51]. While exercise alone improves hepatic steatosis in patients with NAFLD, it does not improve liver fibrosis. Current guidelines recommend a combination of dietary changes with exercise in the management of NAFLD [19].

Diet and weight loss are challenging, especially as regards keeping patients motivated. Usually, patients are told by healthcare providers to make lifestyle changes without being given much support. Most of these challenges arise from lack of behavioral knowledge and motivational techniques among healthcare providers. It is imperative that issues dealing with

Table 3 Pharmacologic treatment options in nonalcoholic steatohepatitis

\begin{tabular}{|c|c|}
\hline Drug & Mechanism of action \\
\hline Orlistat & $\begin{array}{l}\text { Oral inhibitor of gastric and pancreatic } \\
\text { lipases }\end{array}$ \\
\hline Metformin & Oral antihyperglycemic \\
\hline Thiazolidinediones & $\begin{array}{l}\text { Nuclear peroxisome proliferator-activated } \\
\text { receptor- } \gamma \text { agonist }\end{array}$ \\
\hline Liraglutide & Glucagon-like peptide-1 analog \\
\hline Sitagliptin & Dipeptidyl peptidase 4 inhibitor \\
\hline Statin & $\begin{array}{l}\beta \text {-Hydroxy } \beta \text {-methylglutaryl-CoA } \\
\text { reductase inhibitor }\end{array}$ \\
\hline Ezetimibe & Cholesterol-absorption inhibitor \\
\hline $\begin{array}{l}\text { Ursodeoxycholic } \\
\text { acid }\end{array}$ & Bile acid \\
\hline Vitamin E & Antioxidant \\
\hline Obeticholic acid ${ }^{*}$ & Farnesoid X receptor agonist \\
\hline Elafibranor* & $\begin{array}{l}\text { Peroxisome proliferator-activated receptor } \\
\alpha / \delta \text { agonist }\end{array}$ \\
\hline
\end{tabular}

${ }^{\star}$ Ongoing clinical trials weight loss and dietary lifestyle changes are patient-centered and maintained to achieve the desired effects.

\section{Limitations of pharmacologic treatment}

Currently, there are no FDA-approved drugs for treating NASH. However, for some patients, a number of therapeutic options with varying efficacy are available, as listed in Table 3. Pioglitazone compared to placebo in nondiabetic patients showed a favorable improvement in NASH (34\% vs. $19 \%$, $\mathrm{P}=0.04$; number needed to treat $=6.9)[52]$. The same study, the PIVENS (pioglitazone or vitamin E vs. placebo in nondiabetic patients with NASH) trial, showed that the daily use of $800 \mathrm{IU}$ of vitamin E significantly reduced steatosis and inflammation ( $43 \%$ vs. $19 \%, \mathrm{P}=0.001$; number needed to treat $=4.2$ ) [52]. However, some safety concerns have been raised with regard to the use of vitamin $\mathrm{E}$ and pioglitazone; these include postmenopausal bone loss, risk of bladder cancer, hemorrhagic stroke and prostate cancer $[53,54]$. Other medications such as pentoxifylline have shown favorable results, though larger clinical studies are required to evaluate its therapeutic role [55].

Research in the past decade has led to the development of novel therapies. Obeticholic acid, a farnesoid X receptor agonist, was investigated for treating non-cirrhotic NASH patients in the farnesoid X nuclear receptor ligand obeticholic acid for non-cirrhotic, NASH (FLINT) trial. Comparing $25 \mathrm{mg}$ of obeticholic acid per day with placebo, interim analysis showed that the efficacy criterion had been met, leading to discontinuation of the trial. Histological data showed significant improvement in steatohepatitis in $45 \%$ of patients versus $21 \%$ of patients who received placebo [56]. Obeticholic acid was also associated with weight loss. Its potential clinical efficacy and long-term safety are pending the results of a phase III clinical trial (NCT02548351).

Elafibranor, an agonist of the peroxisome proliferatoractivated receptor- $\alpha / \delta$, was recently studied in patients with NASH. The primary outcome of reversal of NASH without worsening of fibrosis was not achieved (19\% vs. $12 \%$; $\mathrm{P}=0.045$ ) [57]. However, using a post-hoc modified definition of NASH resolution, elafibranor $(120 \mathrm{mg} / \mathrm{d}$ for 1 year) versus placebo showed resolution of NASH without worsening fibrosis ( $20 \%$ versus $11 \%, \mathrm{P}=0.018$ ). The use of elafibranor also resulted in improvement in serum lipid levels and liver enzymes. As with obeticholic acid, its potential clinical efficacy and longterm safety are pending the results of a phase III clinical trial (NCT01694849).

Given the increasing prevalence of NAFLD and its consequent public health implications, it is surprising that there are no FDA-recommended therapies. Currently, pioglitazone and vitamin $\mathrm{E}$ are the only recommended therapies according to guidelines in selected patients [58]. However, a joint workshop in 2013 sponsored by the FDA and the AASLD sought to shed light on the gaps hindering progress in this area [59]. The workshop identified a lack of endpoints based on clinical outcomes. Traditionally, all-cause mortality has been the endpoint in therapeutic clinical trials. To 
demonstrate a survival benefit, i.e., improvement in mortality rates, would require a large cohort of patients with early-stage $\mathrm{NASH}$. Additionally, it would require a follow-up period of $10-$ 15 years [59]. NAFLD-related cirrhosis is a slow, progressive disease that takes years to develop. Therefore, such a clinical trial would be costly and logistically challenging.

\section{Surgical therapy and its limitations}

Bariatric surgery in carefully selected patients can be effective in improving NAFLD, as well as other obesity-related comorbidities. A large prospective study with a 5-year follow up showed improvement or reversal of NASH, fibrosis, and NAFLD [60]. Despite these results, bariatric surgery is currently only indicated for the management of obesity and has not been approved as a primary treatment for NAFLD. The cost of the procedure, in addition to its invasiveness, limits its evaluation as a primary treatment modality for NASH. Newly emerging anti-obesity endoscopic procedures, such as intragastric balloon therapy, could play a role in the management of NASH [61]. However, none of these procedures have been clinically evaluated in patients with NAFLD.

\section{Concluding remarks and future directions}

There are many challenges facing patients affected by NAFLD. There are also many challenges faced by clinicians and researchers involved in the management of NAFLD. However, there are opportunities to overcome these challenges. There is increasing evidence that the pathogenesis of NAFLD is guided by an interaction of nutritional habits, genetics, and environmental factors. One area of future research is to explore specific pathways through which these factors interact, especially in the era of epigenetics. A deeper understanding of this complex interaction can lead to the development of guidelines for screening, early diagnosis, and the development of therapeutic strategies. Epigenetic studies will enable us to predict which subset of patients affected by NAFLD are likely to progress into more advanced stages of disease.

Furthermore, the use of noninvasive biomarkers to obviate the need for liver biopsy requires further investigation. Noninvasive staging methods will encourage more patients to enroll in clinical trials, which will further broaden our understanding of the disease. To this end, there is also a need for clinical trials of longer duration to determine clinical endpoints, as well as the long-term safety and durability of new treatment options.

Our review has also shown that there is a need to increase training among healthcare providers with respect to lifestyle behavioral changes and motivational interventions. A patientcentered approach is expected to yield better outcomes in terms of weight loss and its effect on liver fat accumulation. This should also be reflected in authoritative guidelines. The 2012 guidelines of the AASLD need to be updated to reflect current knowledge and trends in order to guide healthcare providers and patients.
NAFLD is emerging as the leading cause of chronic liver disease in the United States and presents an impending social and economic burden. If it is not addressed, a significant portion of healthcare expenditure and resources will be required to cater for NAFLDrelated diseases, further increasing healthcare expenditure. There is a gap in our knowledge and therapeutic armamentarium that needs to be bridged before we can stem the tide.

\section{References}

1. El-Zayadi AR. Hepatic steatosis: a benign disease or a silent killer. World J Gastroenterol 2008;14:4120-4126.

2. Charlton MR, Burns JM, Pedersen RA, Watt KD, Heimbach JK, Dierkhising RA. Frequency and outcomes of liver transplantation for nonalcoholic steatohepatitis in the United States. Gastroenterology 2011;141:1249-1253.

3. Wong RJ, Aguilar M, Cheung R, et al. Nonalcoholic steatohepatitis is the second leading etiology of liver disease among adults awaiting liver transplantation in the United States. Gastroenterology 2015;148:547-555.

4. Musso G, Gambino R, Cassader M, Pagano G. Meta-analysis: natural history of non-alcoholic fatty liver disease (NAFLD) and diagnostic accuracy of non-invasive tests for liver disease severity. Ann Med 2011;43:617-649.

5. Musso G, Gambino R, Tabibian JH, et al. Association of nonalcoholic fatty liver disease with chronic kidney disease: a systematic review and meta-analysis. PLoS Med 2014;11:e1001680.

6. Agarwal AK, Jain V, Singla S, et al. Prevalence of non-alcoholic fatty liver disease and its correlation with coronary risk factors in patients with type 2 diabetes. J Assoc Physicians India 2011;59:351-354.

7. Younossi Z, Anstee QM, Marietti M, et al. Global burden of NAFLD and NASH: trends, predictions, risk factors and prevention. Nat Rev Gastroenterol Hepatol 2018:15:11-20.

8. Said A, Gagovic V, Malecki K, Givens ML, Nieto FJ. Primary care practitioners survey of non-alcoholic fatty liver disease. Ann Hepatol 2013;12:758-765.

9. Younossi ZM, Koenig AB, Abdelatif D, Fazel Y, Henry L, Wymer M. Global epidemiology of nonalcoholic fatty liver diseaseMeta-analytic assessment of prevalence, incidence, and outcomes. Hepatology 2016;64:73-84.

10. Younossi ZM, Stepanova M, Afendy M, et al. Changes in the prevalence of the most common causes of chronic liver diseases in the United States from 1988 to 2008. Clin Gastroenterol Hepatol 2011;9:524-530.

11. Fruci B, Giuliano S, Mazza A, Malaguarnera R, Belfiore A. Nonalcoholic fatty liver: a possible new target for type 2 diabetes prevention and treatment. Int J Mol Sci 2013;14:22933-22966.

12. Byrne CD, Targher G. NAFLD: a multisystem disease. J Hepatol 2015;62:S47-S64.

13. Leite NC, Salles GF, Araujo AL, Villela-Nogueira CA, Cardoso CR. Prevalence and associated factors of non-alcoholic fatty liver disease in patients with type-2 diabetes mellitus. Liver Int 2009;29:113-119.

14. Prashanth M, Ganesh HK, Vima MV, et al. Prevalence of nonalcoholic fatty liver disease in patients with type 2 diabetes mellitus. J Assoc Physicians India 2009;57:205-210.

15. Fan N, Zhang L, Xia Z, Peng L, Wang Y, Peng Y. Sex-specific association between serum uric acid and nonalcoholic fatty liver disease in type 2 diabetic patients. J Diabetes Res 2016;2016:3805372.

16. Sasaki A, Nitta H, Otsuka K, et al. Bariatric surgery and nonalcoholic fatty liver disease: current and potential future treatments. Front Endocrinol (Lausanne) 2014;5:164.

17. Subichin M, Clanton J, Makuszewski M, Bohon A, Zografakis JG, 
Dan A. Liver disease in the morbidly obese: a review of 1000 consecutive patients undergoing weight loss surgery. Surg Obes Relat Dis 2015;11:137-141.

18. Williams CD, Stengel J, Asike MI, et al. Prevalence of nonalcoholic fatty liver disease and nonalcoholic steatohepatitis among a largely middle-aged population utilizing ultrasound and liver biopsy: a prospective study. Gastroenterology 2011;140:124-131.

19. Chalasani N, Younossi Z, Lavine JE, et al. The diagnosis and management of nonalcoholic fatty liver disease: Practice guidance from the American Association for the Study of Liver Diseases. Hepatology 2017:67:328-357.

20. Younossi ZM, Zheng L, Stepanova M, Henry L, Venkatesan C, Mishra A. Trends in outpatient resource utilizations and outcomes for Medicare beneficiaries with nonalcoholic fatty liver disease. J Clin Gastroenterol 2015;49:222-227.

21. Sayiner M, Otgonsuren M, Cable R, et al. Variables associated with inpatient and outpatient resource utilization among Medicare beneficiaries with nonalcoholic fatty liver disease with or without cirrhosis. J Clin Gastroenterol 2017;51:254-260.

22. Chawla KS, Talwalkar JA, Keach JC, Malinchoc M, Lindor KD, Jorgensen R. Reliability and validity of the Chronic Liver Disease Questionnaire (CLDQ) in adults with non-alcoholic steatohepatitis (NASH). BMJ Open Gastroenterol 2016;3:e000069.

23. Calzadilla Bertot L, Adams LA. The natural course of non-alcoholic fatty liver disease. Int J Mol Sci 2016;17.

24. Weiß J, Rau M, Geier A. Non-alcoholic fatty liver disease: epidemiology, clinical course, investigation, and treatment. Dtsch Arztebl Int 2014;111:447-452.

25. Ekstedt M, Hagström $H$, Nasr $P$, et al. Fibrosis stage is the strongest predictor for disease-specific mortality in NAFLD after up to 33 years of follow-up. Hepatology 2015;61:1547-1554.

26. White DL, Kanwal F, El-Serag H. Association between nonalcoholic fatty liver disease and risk for hepatocellular cancer, based on systematic review. Clin Gastroenterol Hepatol 2012;10:1342-1359.e2.

27. Mittal S, Sada YH, El-Serag HB, et al. Temporal trends of nonalcoholic fatty liver disease-related hepatocellular carcinoma in the veteran affairs population. Clin Gastroenterol Hepatol 2015;13:594-601.

28. Angulo P, Kleiner D, Dam-Larsen S, et al. The prognostic relevance of liver histology features in nonalcoholic fatty liver disease: the PRELHIN study. Hepatology 2014;60:226A-227A.

29. Bergqvist CJ, Skoien R, Horsfall L, Clouston AD, Jonsson JR, Powell EE. Awareness and opinions of non-alcoholic fatty liver disease by hospital specialists. Intern Med J 2013;43:247-253.

30. Ratziu V, Cadranel JF, Serfaty L, et al. A survey of patterns of practice and perception of NAFLD in a large sample of practicing gastroenterologists in France. J Hepatol 2012;57:376-383.

31. Fracanzani AL, Valenti L, Bugianesi E, et al. Risk of severe liver disease in nonalcoholic fatty liver disease with normal aminotransferase levels: a role for insulin resistance and diabetes. Hepatology 2008;48:792-798.

32. Vos MB, Abrams SH, Barlow SE, et al. NASPGHAN clinical practice guideline for the diagnosis and treatment of nonalcoholic fatty liver disease in children: recommendations from the Expert Committee on NAFLD (ECON) and the North American Society of Pediatric Gastroenterology, Hepatology and Nutrition (NASPGHAN). J Pediatr Gastroenterol Nutr 2017;64:319-334.

33. National Guideline Centre (UK). Non-alcoholic fatty liver disease: assessment and management. National Institute for Health and Care Excellence: Guidance 2016.

34. European Association for the Study of Obesity (EASO). EASLEASD-EASO clinical practice guidelines for the management of non-alcoholic fatty liver disease. Obes Facts 2016;9:65-90.

35. Lee SS, Park SH. Radiologic evaluation of nonalcoholic fatty liver disease. World J Gastroenterol 2014;20:7392-7402.

36. Lee DH. Imaging evaluation of non-alcoholic fatty liver disease: focused on quantification. Clin Mol Hepatol 2017:23:290-301.

37. Schwenzer NF, Springer F, Schraml C, Stefan N, Machann J, Schick F. Non-invasive assessment and quantification of liver steatosis by ultrasound, computed tomography and magnetic resonance. J Hepatol 2009;51:433-445.

38. Angulo P, Hui JM, Marchesini G, et al. The NAFLD fibrosis score: a noninvasive system that identifies liver fibrosis in patients with NAFLD. Hepatology 2007;45:846-854.

39. Angulo P, Kleiner DE, Dam-Larsen S, et al. Liver fibrosis, but no other histologic features, associates with long-term outcomes of patients with nonalcoholic fatty liver disease. Gastroenterology 2015;149:389-397.e10.

40. Kaswala DH, Lai M, Afdhal NH. Fibrosis assessment in nonalcoholic fatty liver disease (NAFLD) in 2016. Dig Dis Sci 2016;61:1356-1364.

41. Festi D, Schiumerini R, Marzi L, et al. Review article: the diagnosis of non-alcoholic fatty liver disease - availability and accuracy of non-invasive methods. Aliment Pharmacol Ther 2013;37:392-400.

42. Tapper EB, Challies T, Nasser I, Afdhal NH, Lai M. The performance of vibration controlled transient elastography in a US cohort of patients with non-alcoholic fatty liver disease. Am J Gastroenterol 2016;111:677-684.

43. Machado MV, Cortez-Pinto $H$. Non-invasive diagnosis of non-alcoholic fatty liver disease. A critical appraisal. $J$ Hepatol 2013;58:1007-1019.

44. Loomba R, Wolfson T, Ang B, et al. Magnetic resonance elastography predicts advanced fibrosis in patients with nonalcoholic fatty liver disease: a prospective study. Hepatology 2014;60:1920-1928.

45. Vilar-Gomez E, Martinez-Perez Y, Calzadilla-Bertot L, et al. Weight loss via lifestyle modification significantly reduces features of nonalcoholic steatohepatitis. Gastroenterology 2015; 149:367-378.e5.

46. Wong VW, Chan RS, Wong GL, et al. Community-based lifestyle modification programme for non-alcoholic fatty liver disease: a randomized controlled trial. J Hepatol 2013;59:536-542.

47. Katsagoni CN, Georgoulis M, Papatheodoridis GV, Panagiotakos DB, Kontogianni MD. Effects of lifestyle interventions on clinical characteristics of patients with non-alcoholic fatty liver disease: a meta-analysis. Metabolism 2017;68:119-132.

48. Ryan MC, Itsiopoulos C, Thodis T, et al. The Mediterranean diet improves hepatic steatosis and insulin sensitivity in individuals with non-alcoholic fatty liver disease. J Hepatol 2013;59:138-143.

49. Katsagoni CN, Papatheodoridis GV, Papageorgiou MV, et al. A "healthy diet-optimal sleep" lifestyle pattern is inversely associated with liver stiffness and insulin resistance in patients with nonalcoholic fatty liver disease. Appl Physiol Nutr Metab 2017;42:250-256.

50. Katsagoni CN, Georgoulis M, Papatheodoridis GV, et al. Associations between lifestyle characteristics and the presence of nonalcoholic fatty liver disease: a case-control study. Metab Syndr Relat Disord 2017;15:72-79.

51. Oh S, Shida T, Yamagishi K. Moderate to vigorous physical activity volume is an important factor for managing nonalcoholic fatty liver disease: a retrospective study. Hepatology 2015;61:1205-1215.

52. Sanyal AJ, Chalasani N, Kowdley KV, et al; NASH CRN. Pioglitazone, vitamin E, or placebo for nonalcoholic steatohepatitis. N Engl J Med 2010;362:1675-1685.

53. Lewis JD, Ferrara A, Peng T, et al. Risk of bladder cancer among diabetic patients treated with pioglitazone: interim report of a longitudinal cohort study. Diabetes Care 2011;34:916-922.

54. Gee PT. Unleashing the untold and misunderstood observations on vitamin E. Genes Nutr 2011;6:5-16.

55. Van Wagner LB, Koppe SW, Brunt EM, et al. Pentoxifylline for 
the treatment of non-alcoholic steatohepatitis: a randomized controlled trial. Ann Hepatol 2011;10:277-286.

56. Neuschwander-Tetri BA, Loomba R, Sanyal AJ, et al; NASH Clinical Research Network. Farnesoid X nuclear receptor ligand obeticholic acid for non-cirrhotic, non-alcoholic steatohepatitis (FLINT): a multicentre, randomised, placebo-controlled trial. Lancet 2015;385:956-965.

57. Ratziu V, Harrison SA, Francque S, et al.; GOLDEN-505 Investigator Study Group. Elafibranor, an agonist of the peroxisome proliferator-activated receptor-alpha and -delta, induces resolution of nonalcoholic steatohepatitis without fibrosis worsening. Gastroenterology 2016;150:1147-1159.

58. Lazaridis N, Tsochatzis E. Current and future treatment options in non-alcoholic steatohepatitis (NASH). Expert Rev Gastroenterol
Hepatol 2017;11:357-369.

59. Sanyal AJ, Friedman SL, McCullough AJ, Dimick-Santos L; United States Food and Drug Administration. Challenges and opportunities in drug and biomarker development for nonalcoholic steatohepatitis: findings and recommendations from an American Association for the Study of Liver Diseases-U.S. Food and Drug Administration Joint Workshop. Hepatology 2015;61:1392-1405.

60. Taitano AA, Markow M, Finan JE, Wheeler DE, Gonzalvo JP, Murr MM. Bariatric surgery improves histological features of nonalcoholic fatty liver disease and liver fibrosis. J Gastrointest Surg 2015; 19:429-436.

61. Abu Dayyeh BK, Edmundowicz SA, Jonnalagadda S, et al; ASGE Technology Committee. Endoscopic bariatric therapies. Gastrointest Endosc 2015;81:1073-1086. 